\title{
Abnormal Sonic hedgehog signaling in the lung of rats with esophageal atresia induced by adriamycin
}

\author{
Ana Catarina Fragoso ${ }^{1-3}$, Leopoldo Martinez ${ }^{1,2}$, José Estevão-Costa ${ }^{3}$ and Juan A. Tovar ${ }^{1,2}$
}

BACKGROUND: Abnormal lung development was recently described in the rat model of esophageal atresia and tracheoesophageal fistula (EA-TEF). Since in this condition the ventral-to-dorsal switch of Shh expression in the foregut is disturbed, the present study tested the hypothesis that this abnormal expression at the emergence of the tracheobronchial bud might be translated into the developing lung.

METHODS: Pregnant rats received either $1.75 \mathrm{mg} / \mathrm{kg}$ i.p. adriamycin or vehicle from E7 to E9. Three groups were studied: control and adriamycin-exposed with and without EA-TEF. Embryos were recovered and the lungs were harvested and processed for reverse transcription polymerase chain reaction and immunofluorescence analysis of the Shh signaling cascade.

RESULTS: Shh signaling was downregulated at the late embryonic stage of lung development (E13) in embryos with EA-TEF. Throughout the subsequent stages of development, the expression of both Shh and its downstream components increased significantly and remained upregulated throughout gestation. Immunofluorescent localization was consistent with these findings.

CONCLUSION: Defective Shh signaling environment in the foregut is present beyond the emergence of lung buds and probably impairs lung development. Later in gestation, lungs exhibited a remarkable ability to upregulate the Shh cascade, suggesting a compensatory response. These findings may be relevant to understand pulmonary disease suffered by children with EA-TEF.

$\mathbf{P}$ renatal exposure to adriamycin consistently induces esophageal atresia with tracheoesophageal fistula (EA-TEF) and other anomalies of the VACTERL (Vertebral, Anal, Cardiac, TracheoEsophageal, Renal, and Limb) association in rats and mice. These toxicologic models involve abnormal notochord positioning, volume, or branching. The notochord plays a critical role in the inductive interaction that patterns the tissues of the neural tube, the paraxial mesoderm, and the endodermal derivatives of the gut, namely foregut organization and division (1). Several reports demonstrated that adriamycinexposed rodent embryos with EA-TEF have a deformed $(2,3)$, hypertrophic (4), abnormally branched (5), or ectopically located notochord (1).

Both the digestive and respiratory systems derive from a common embryonic organ, the foregut. The respiratory system originates from an endodermal diverticulum in the ventral wall of the foregut whereas the esophagus derives from the dorsal wall. Because of this common origin, abnormal play of the factors that modulate the development often results in structural anomalies involving both systems (6).

The inductive activity of the notochord is mediated by the morphogene Sonic hedgehog $(S h h)$. The Hh pathway regulates foregut development and lung morphogenesis through specific and dynamic epithelial-mesenchymal interactions and the Shh signal is initially expressed in the notochord, foregut, and ventral part of the neural tube (1). The Shh gene encodes a secreted intercellular signaling polypeptide that binds to a transmembrane receptor complex comprising two components: Patched (Ptch), a negative regulator, and Smoothened (Smo), a positive transducer of Shh signaling. Shh binding to Ptch releases Smo that will activate three zinc finger transcription factors Gli1, Gli2, and Gli3 that regulate the transcription of $S h h$-responsive target genes (7). In studies with Shh pathway mutant mice, the spectrum of foregut malformations ranges from esophageal and tracheal stenosis and lung dysplasia to esophageal atresia and tracheoesophageal fistula with severe lung hypoplasia; moreover, the disturbance in Shh signal transduction as seen in $\mathrm{Gli2}^{-/}$; $\mathrm{Gli3}^{+} /^{-}$mice results in a similar $\mathrm{Shh}^{-/^{-}}$phenotype whereas Gli2 ${ }^{--}$; Gli3 $^{-/^{-}}$mice present more severe characteristics illustrating the importance of gene dosage in the Shh pathway during foregut development $(7,8)$.

In the adriamycin toxicologic model of esophageal atresia, the ectopic localization of the notochord induced by adriamycin leads to disruption in Shh signaling in the prospective site of tracheoesophageal separation in the rat embryo $(1,9)$. Moreover, the level of Shh protein expression in the foregut is much lower than in control embryos and does not show timedependent changes (10). Shh expression in the mouse respiratory primordium has an important role in lung branching and its misexpression results in severe alveolar hypoplasia and a significant increase in lung mesenchyme (11). 


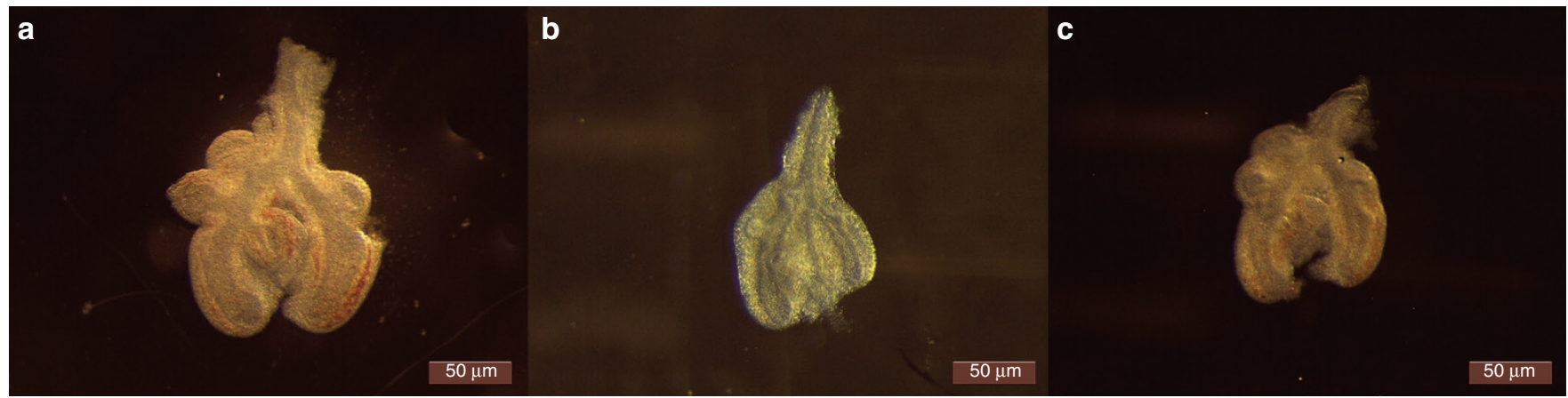

Figure 1. Lung mass at the embryonic stage of development (E13). (a) Control; (b) adria EA-TEF (esophageal atresia and tracheoesophageal fistula); (c) adria noEA. Smaller lungs were observed in both adriamycin-exposed groups. The adria EA-TEF group exhibited the smallest size, suggesting a detrimental and additive effect of the malformation on growth of adriamycin-exposed lungs (original magnification: $\times 40$ ).

In the human condition this implication is still speculative. Nevertheless, mutations/deletions affecting FOXF1, which is linked to Shh signaling, and mutation of HOXD13, a downstream target of $S h$, result in a VACTERL-like phenotype (12); furthermore, several human syndromic esophageal atresias implicate deletions/mutations in genes that also seem to interact with the Shh pathway as MYCN (Feingold syndrome) $(13,14)$, SOX2 (anophthalmia-esophageal-genital syndrome) $(15,16)$, Mid1 (X-linked Opitz syndrome) $(17,18)$, and Gli3 (Pallister-Hall syndrome) $(19,20)$. These reported genetic disorders suggest that disturbances of Shh signaling due to interacting pathways may play a role in the EA-TEF/VACTERL association phenotype. Along with the gastrointestinal phenotype, defects in signaling via Shh are associated with pulmonary malformations such as lung hypoplasia of varying levels of severity as seen in Pallister-Hall and Smith-Lemli-Opitz syndromes or in the VACTERL association itself (21).

Adriamycin-induced EA-TEF is associated with defective tracheobronchial branching and some degree of lung hypoplasia $(22,23)$. The present study examined whether the abnormal foregut expression pattern of Shh at the site of emergence of the tracheobronchial bud is translated into the developing lung of rats with EA-TEF/VACTERL association. For this purpose, the messenger RNA of several components of the cascade genes involved in Shh regulation was measured by real-time reverse transcription polymerase chain reaction (RT-PCR) and their respective proteins were immunolocalized in the lungs at specific time endpoints, embryonic days $13,15,18$, and 21 (E13, E15, E18, and E21) corresponding to the late embryonic, pseudoglandular, canalicular, and saccular stages of lung development.

\section{RESULTS}

Lung Mass at the Embryonic Stage of Development

Adriamycin-exposed rats had smaller lungs than controls, and this feature was more marked in those with EA-TEF. This is depicted in Figure 1.

\section{Messenger RNA Expression of Genes of the Shh Signaling Pathway}

These results are shown in Figures 2-6.

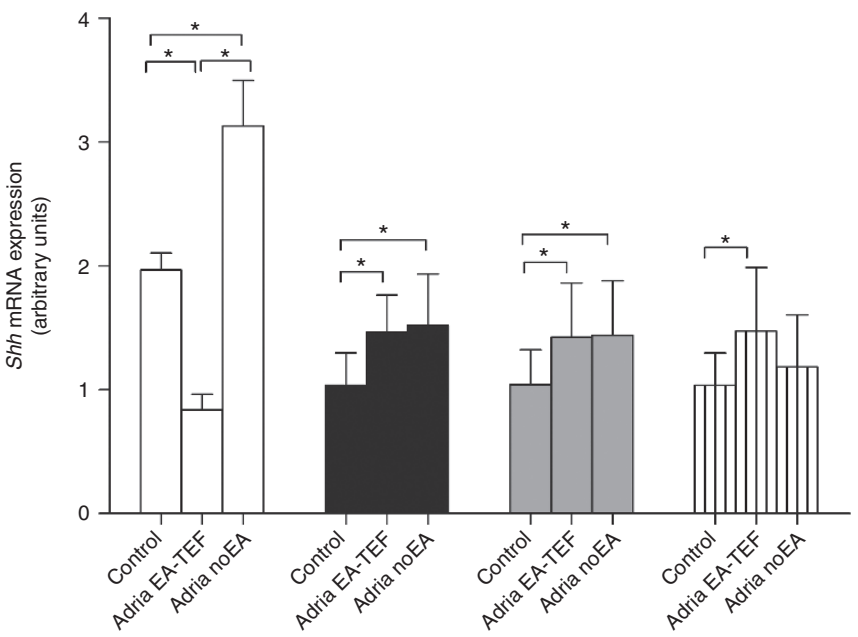

Figure 2. Shh mRNA expression at the late embryonic, pseudoglandular, canalicular, and saccular stages of lung development. Lungs from adriamycin-exposed with EA-TEF (esophageal atresia and tracheoesophageal fistula) fetuses showed a significantly lower expression in contrast to the adria noEA group that exhibited the highest levels, at E13 (white bars). From E13 onwards, Shh mRNA was overexpressed by both adriamycinexposed lungs with the exception of adria noEA at E21 (hatched bars), whose expression level was similar to control lungs. E13: control vs. adria EA-TEF ${ }^{*} P<0.001$; control vs. adria noEA $* P<0.01$; adria EA-TEF vs. adria noEA ${ }^{*} P<0.001$ (control $(n=3): 1.976 \pm 0.136$; adria $\operatorname{EA-TEF}(n=3)$ : $0.834 \pm 0.125$; adria noEA ( $n=3)$ : $3.130 \pm 0.367)$; E15 (black bars): control vs. adria EA-TEF $* P<0.01$; control vs. adria noEA $* P=0.01$; adria EA-TEF vs. adria noEA $P$ value is not significant (control $(n=13)$ : $1.035 \pm 0.262$; adria $\operatorname{EA}-\operatorname{TEF}(n=9): 1.463 \pm 0.3$; adria noEA $(n=13)$ : $1.519 \pm 0.416)$; E18 (gray bars): control vs. adria EA-TEF $* P=0.02$; control vs. adria noEA $* P$ $=0.02$; adria EA-TEF vs. adria noEA $P$ value is not significant (control $(n=$ 10): $1.039 \pm 0.284$; adria EA-TEF $(n=11): 1.424 \pm 0.438$; adria noEA $(n=10)$; $1.437 \pm 0.442$ ); E21: control vs. adria EA-TEF ${ }^{*} P=0.04$; control vs. adria noEA $P$ value is not significant; adria EA-TEF vs. adria noEA $P$ value is not significant (control $(n=12)$ : $1.034 \pm 0.263$; adria EA-TEF $(n=12): 1.474 \pm 0.513$; adria noEA $(n=11): 1.184 \pm 0.420)$.

\section{E13 (Late Embryonic Stage)}

The lungs of rat embryos with EA-TEF induced by adriamycin exhibited significantly lower levels of mRNA of all studied components of the Shh signaling pathway. In contrast, the lungs of adriamycin-exposed embryos without EA-TEF showed the highest mRNA levels except for Ptch. 


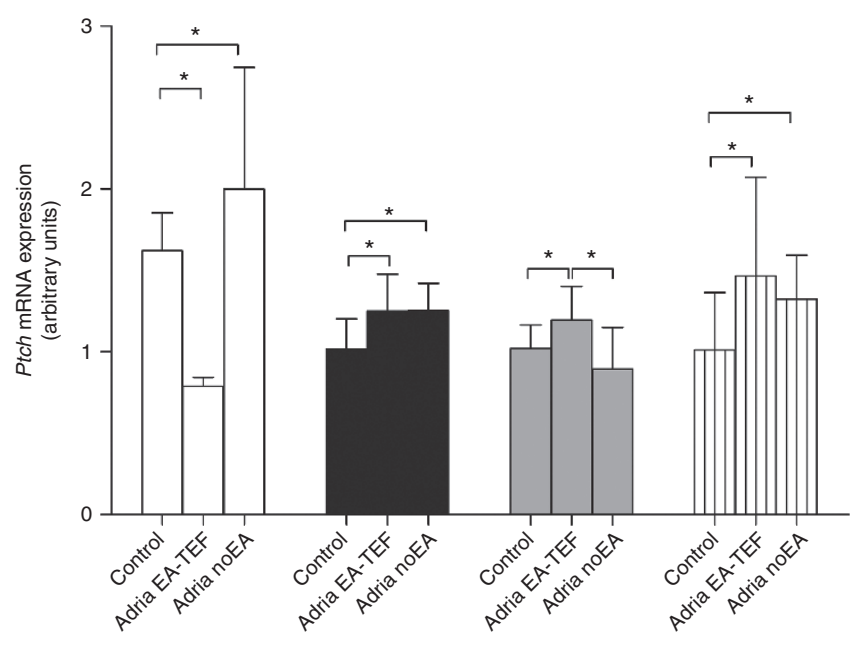

Figure 3. Ptch mRNA expression at the late embryonic, pseudoglandular, canalicular, and saccular stages of lung development. At the embryonic stage of lung development (E13, white bars), lungs from adriamycinexposed with EA-TEF (esophageal atresia and tracheoesophageal fistula) showed significantly lower levels of Ptch mRNA expression. From E13 onwards, a consistent overexpression in both adriamycin-exposed lungs was observed with the exception of adria noEA at E18 (gray bars), whose expression level was similar to control lungs. E13: control vs. adria EA-TEF ${ }^{*} P<0.01$; control vs. adria noEA $P$ value is not significant; adria EA-TEF vs. adria noEA ${ }^{*} P=0.04$ (control $(n=3)$ : $1.621 \pm 0.234$; adria $\operatorname{EA}-\mathrm{TEF}(n=3)$ : $0.785 \pm 0.056$; adria noEA $(n=3): 1.999 \pm 0.748)$; E15 (black bars): control vs. adria EA-TEF $* P=0.01$; control vs. adria noEA $* P<0.01$; adria EA-TEF vs. adria noEA $P$ value is not significant (control $(n=13): 1.016 \pm 0.187$; adria $\operatorname{EA}-\operatorname{TEF}(n=9)$ : $1.249 \pm 0.227$; adria noEA $(n=13): 1.252 \pm 0.168)$; E18: control vs. adria EA-TEF $* P=0.04$; control vs. adria noEA $P$ value is not significant; adria EA-TEF vs. adria noEA $* P=0.01$ (control $(n=10)$ : 1.019 \pm 0.146 ; adria EA-TEF $(n=10)$ : $1.195 \pm 0.207$; adria noEA $(n=9): 0.893 \pm 0.256)$; E21 (hatched bars): control vs. adria EA-TEF ${ }^{*} P=0.03$; control vs. adria noEA $* P$ $=0.02$; adria EA-TEF vs. adria noEA $P$ value is not significant (control $(n=$ 12): $1.010 \pm 0.354$; adria EA-TEF $(n=13): 1.466 \pm 0.607$; adria noEA $(n=11)$ : $1.325 \pm 0.268)$.

\section{E15 (Pseudoglandular Stage)}

The lungs from the adria EA-TEF group of fetuses significantly overexpressed Shh, Ptch, Smo, Gli2, and Gli3 mRNA, and so did fetuses from the adria noEA group with the exception of Gli3 levels that were similar to control.

\section{E18 (Canalicular Stage)}

A significant upregulation of all the components of the Shh signaling cascade was demonstrated in the lungs of adria EA-TEF fetuses. In contrast, in the adria noEA group only the expression of Shh mRNA was significantly upregulated. Gli3 mRNA levels were significantly lower than controls. When comparing the lungs of both adriamycin-exposed groups, the levels of Ptch, Smo, and Gli2 were significantly higher in animals with EA-TEF than in those without it.

\section{E21 (Saccular Stage)}

Near the end of gestation, the lungs of adria EA-TEF fetuses expressed significantly higher levels of every Shh signaling cascade player and so did the lungs of the adria noEA fetuses, with the exception of Shh mRNA whose levels were similar to those of controls.

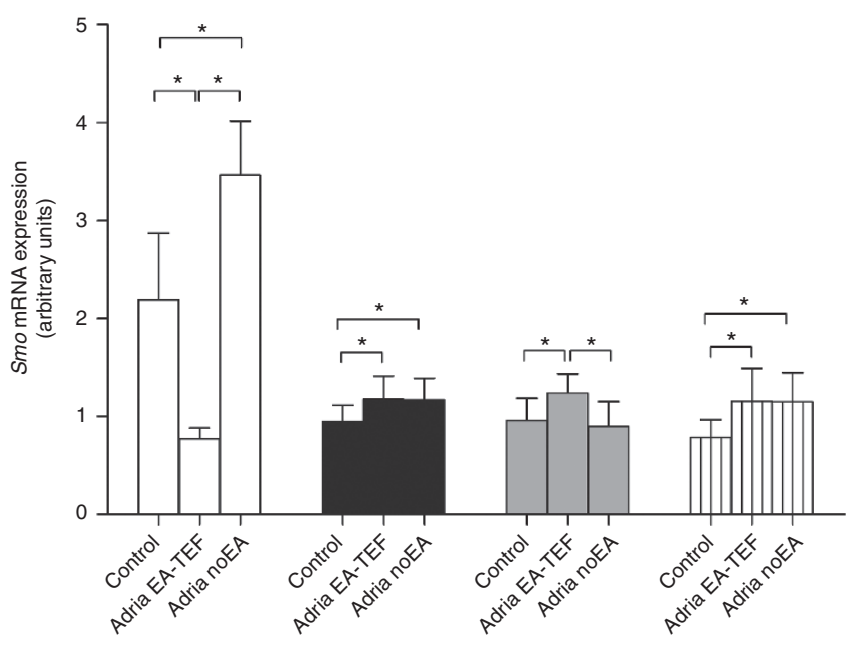

Figure 4. Smo mRNA expression at the late embryonic, pseudoglandular, canalicular, and saccular stages of lung development. At E13 (white bars), adria EA-TEF (esophageal atresia and tracheoesophageal fistula) lungs showed a significantly lower expression level in contrast to the adria noEA group that overexpressed Smo mRNA. Once again, both adriamycinexposed lungs exhibited a significantly higher expression level during the rest of gestation with the exception of adria noEA lungs at E18 (gray bars). E13: control vs. adria EA-TEF ${ }^{*} P=0.02$; control vs. adria noEA ${ }^{*} P=$ 0.04 ; adria EA-TEF vs. adria noEA $* P=0.01$ (control $(n=3): 2.158 \pm 0.647$; adria $\operatorname{EA}-\operatorname{TEF}(n=3): 0.772 \pm 0.109$; adria noEA $(n=3): 3.563 \pm 0.537)$; E15 (black bars): control vs. adria EA-TEF $* P=0.04$; control vs. adria noEA $* P$ $=0.03$; adria EA-TEF vs. adria noEA $P$ value is not significant (control $(n=$ 8): $0.950 \pm 0.163$; adria EA-TEF $(n=8)$ : $1.176 \pm 0.235$; adria noEA $(n=10)$ : $1.170 \pm 0.218$ ); E18: control vs. adria EA-TEF ${ }^{*} P=0.01$; control vs. adria noEA $P$ value is not significant; adria EA-TEF vs. adria noEA * $P<0.01$ (control ( $n$ =9): $0.959 \pm 0.227$; adria $\operatorname{EA}-\operatorname{TEF}(n=8): 1.239 \pm 0.194$; adria noEA $(n=9)$ : $0.899 \pm 0.252$ ); E21 (hatched bars): control vs. adria EA-TEF ${ }^{*} P=0.01$; control vs. adria noEA $* P<0.01$; adria EA-TEF vs. adria noEA $P$ value is not significant (control $(n=9)$ : $0.785 \pm 0.180$; adria EA-TEF $(n=10): 1.155 \pm 0.335$; adria noEA ( $n=10): 1.148 \pm 0.299)$.

\section{Immunofluorescence Distribution}

The lungs from control, adria EA-TEF, and adria noEA groups were at the same stage of lung development at the specific time endpoints selected.

Clear immunoreactivity was detected for Shh, Ptch, Smo, Gli2, and Gli3 at the pseudoglandular (E15), canalicular (E18), and saccular stages (E21) of lung development. All antibodies were mainly expressed in the epithelium, although Smo, Gli2, and Gli3 also exhibited disperse immunoreactivity throughout the surrounding mesenchyme.

\section{Lungs From Control Fetuses}

All the studied proteins were detected at the three stages of lung development with a predominantly epithelial immunolocalization (Figures 7-9).

\section{Lungs From Fetuses Exposed to Adriamycin With EA-TEF}

At the three time endpoints selected, E15, E18, and E21, these lungs appeared to express increased epithelial immunoreactivity for Shh and its downstream components. The epithelial and mesenchymal localization were similar to those of control lungs (Figures 7-9). 


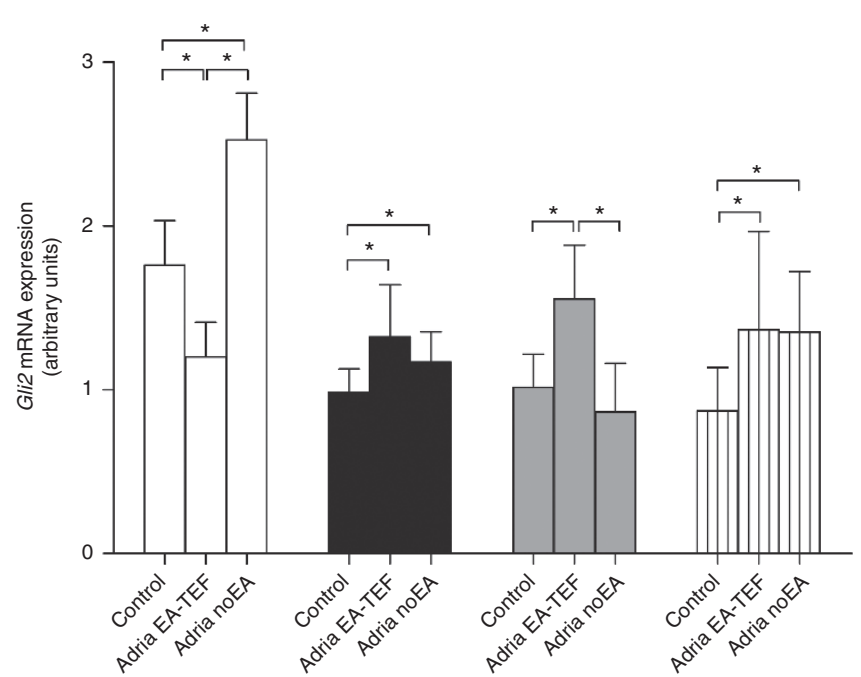

Figure 5. Gli2 mRNA expression at the late embryonic, pseudoglandular, canalicular, and saccular stages of lung development. Adria EA-TEF (esophageal atresia and tracheoesophageal fistula) lungs showed a significantly lower level of Gli2 mRNA in contrast to the highest level expressed by the adria noEA group at the late embryonic stage of development (white bars). Once more, from E13 onwards consistent higher levels of Gli2 mRNA expression were observed in both adriamycin-exposed lungs except for the adria noEA group at E18 (gray bars). E13: control vs. adria EA-TEF ${ }^{*} P=$ 0.04 ; control vs. adria noEA $* P=0.02$; adria EA-TEF vs. adria noEA $* P<0.01$ (control $(n=3)$ : $1.761 \pm 0.271$; adria EA-TEF $(n=3)$ : $1.2 \pm 0.213$; adria noEA $(n=3): 2.525 \pm 0.286)$; E15 (black bars): control vs. adria EA-TEF $* P=0.04$; control vs. adria noEA $* P=0.03$; adria EA-TEF vs. adria noEA $P$ value is not significant (control $(n=9): 0.985 \pm 0.142$; adria EA-TEF $(n=9)$ : $1.322 \pm 0.319$; adria noEA $(n=8)$ : $1.169 \pm 0.186)$; E18: control vs. adria EA-TEF ${ }^{*} P=0.001$; control vs. adria noEA $P$ value is not significant; adria EA-TEF vs. adria noEA ${ }^{*} P<0.001$ (control $(n=9)$ : $1.014 \pm 0.204$; adria EA-TEF $(n=7): 1.553 \pm 0.330$; adria noEA $(n=9): 0.865 \pm 0.295)$; E21 (hatched bars): control vs. adria EA-TEF $* P=0.01$; control vs. adria noEA $* P<0.01$; adria EA-TEF vs. adria noEA $P$ value is not significant (control $(n=9)$ : $0.785 \pm 0.180$; adria EA-TEF $(n=10): 1.155 \pm 0.335 ;$ adria noEA $(n=10): 1.148 \pm 0.299)$.

\section{Lungs From Fetuses Exposed to Adriamycin Without EA-TEF}

At E15, immunoreactivity for Shh, Ptch, Smo, and Gli2 was apparently similar in the lungs of these fetuses and those with EA-TEF. Gli3 protein was less visible in the mesenchyme, as in control lungs. At E18, immunoreactivity for Shh was increased as in the lungs of fetuses with EA-TEF whereas Gli3 seemed decreased; Ptch, Smo, and Gli2 remained in levels comparable to those of the control group. At E21, all but Shh proteins were slightly overexpressed in adria noEA lungs (Figures 7-9).

\section{DISCUSSION}

Lung development has a broad impact in the practice of pediatricians and pediatric surgeons involved in the treatment of children with congenital lung malformations, congenital diaphragmatic hernia, esophageal atresia, and tracheoesophageal fistula that are often accompanied by lung hypoplasia and/or prematurity or followed by chronic respiratory morbidity.

It was recently reported that rat fetuses with esophageal atresia and tracheoesophageal fistula induced by adriamycin have hypoplastic lungs and abnormal control of branching with FGF10 (fibroblast growth factor 10) overexpression in the

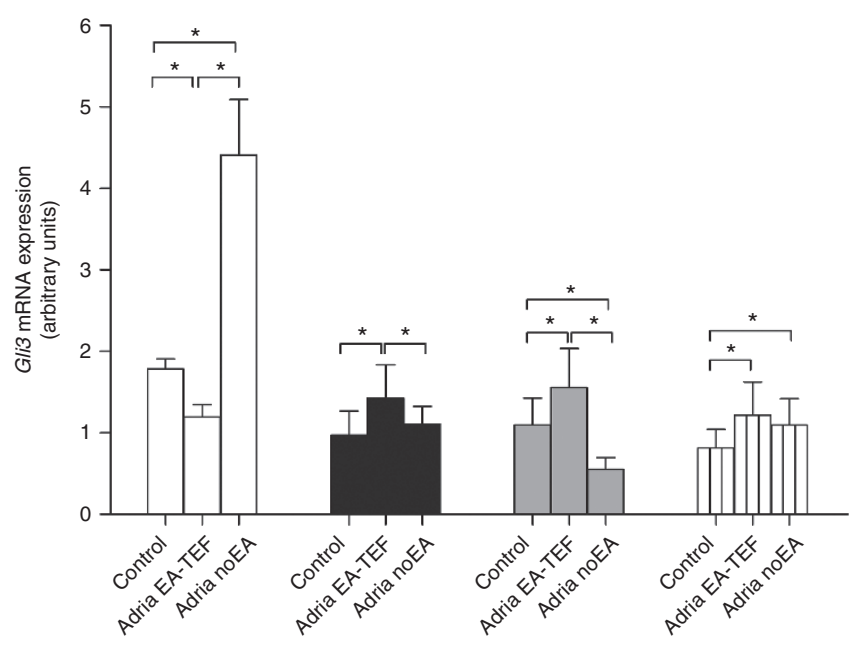

Figure 6. Gli3 mRNA expression at the late embryonic, pseudoglandular, canalicular, and saccular stages of lung development. At the earliest studied stage of lung development (E13, white bars), Gli3 mRNA was underexpressed by adria EA-TEF (esophageal atresia and tracheoesophageal fistula) lungs in contrast to the overexpression observed in the adria noEA group. From E13 onwards, both adriamycin-exposed lungs showed significantly higher levels of expression with the exception of adria noEA at E15 (black bars), whose expression level was similar to control lungs. E13: control vs. adria EA-TEF ${ }^{*} P<0.01$; control vs. adria noEA ${ }^{*} P=0.01$; adria EA-TEF vs. adria noEA $* P=0.001$ (control $(n=3): 1.785 \pm 0.122$; adria $\operatorname{EA}-\mathrm{TEF}(n=3)$ : $1.198 \pm 0.150$; adria noEA $(n=3): 4.408 \pm 0.685)$; E15: control vs. adria EA-TEF ${ }^{*} P=0.01$; control vs. adria noEA $P$ value is not significant; adria EA-TEF vs. adria noEA $P$ value is not significant (control ( $n$ $=9): 0.972 \pm 0.295$; adria $\operatorname{EA}-\mathrm{TEF}(n=8): 1.430 \pm 0.405$; adria noEA $(n=9)$ : $1.109 \pm 0.216$ ); E18 (gray bars): control vs. adria EA-TEF ${ }^{*} P=0.02$; control vs. adria noEA * $P<0.01$; adria EA-TEF vs. adria noEA $* P<0.0001$ (control ( $n$ $=10): 1.096 \pm 0.329$; adria $\operatorname{EA}-\operatorname{TEF}(n=8): 1.556 \pm 0.481$; adria noEA $(n=9)$ : $0.551 \pm 0.148$ ); $E 21$ (hatched bars): control vs. adria EA-TEF $* P=0.01$; control vs. adria noEA $* P=0.04$; adria EA-TEF vs. adria noEA $P$ value is not significant (control $(n=9)$ : $0.817 \pm 0.228$; adria EA-TEF $(n=11): 1.220 \pm 0.406$; adria noEA $(n=10): 1.096 \pm 0.323)$.

pseudoglandular, canalicular, and saccular stage of lung development $(22,23)$.

In adriamycin-exposed embryos, the emergence of the respiratory component is abnormal because the esophagotracheal foregut tube fails to divide (24). The abnormal emergence of the lung bud at E12 seems to affect lung growth. In fact, our results revealed that at the embryonic stage of lung development (E13) in the adriamycin-exposed embryos the lungs are smaller than controls with those with EA-TEF showing the most marked reduction of lung mass and downregulation of the Shh pathway, suggesting a previously defective Shh environment in the adriamycin-exposed embryos further exacerbated by the malformation itself. From the pseudoglandular stage onwards, a remarkable switch in Shh expression levels and its downstream pathway components takes place and a consistent overexpression of Shh, Ptch, Smo, Gli2, and Gli3 mRNA was observed in comparison to the control lungs. It suggests the presence of an intrinsic mechanism pointing to recovery of normal growth since Shh overexpression in the developing lung leads to enhanced epithelial and mesenchymal cell proliferation (25). An interesting finding in the adriamycin-exposed rats without EA-TEF was that Shh signaling pathway levels of expression 

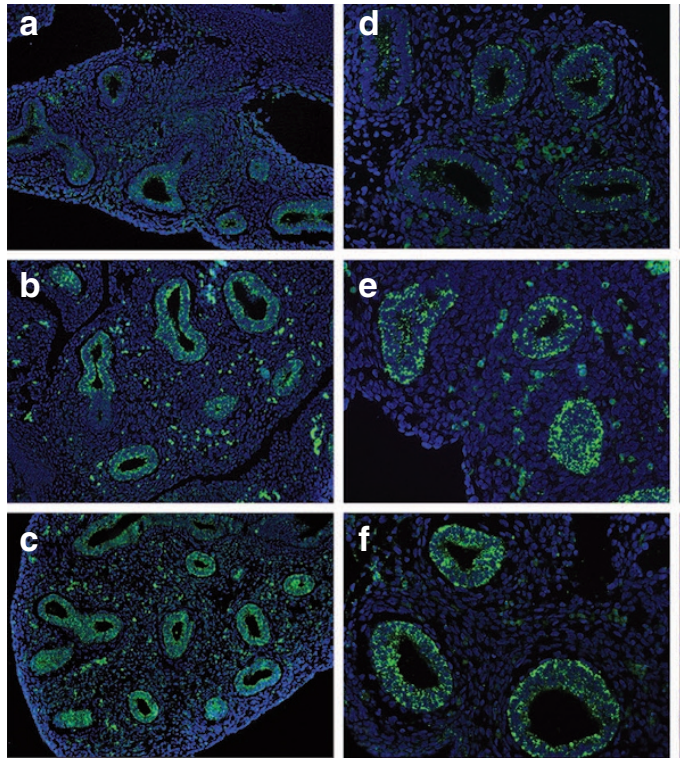
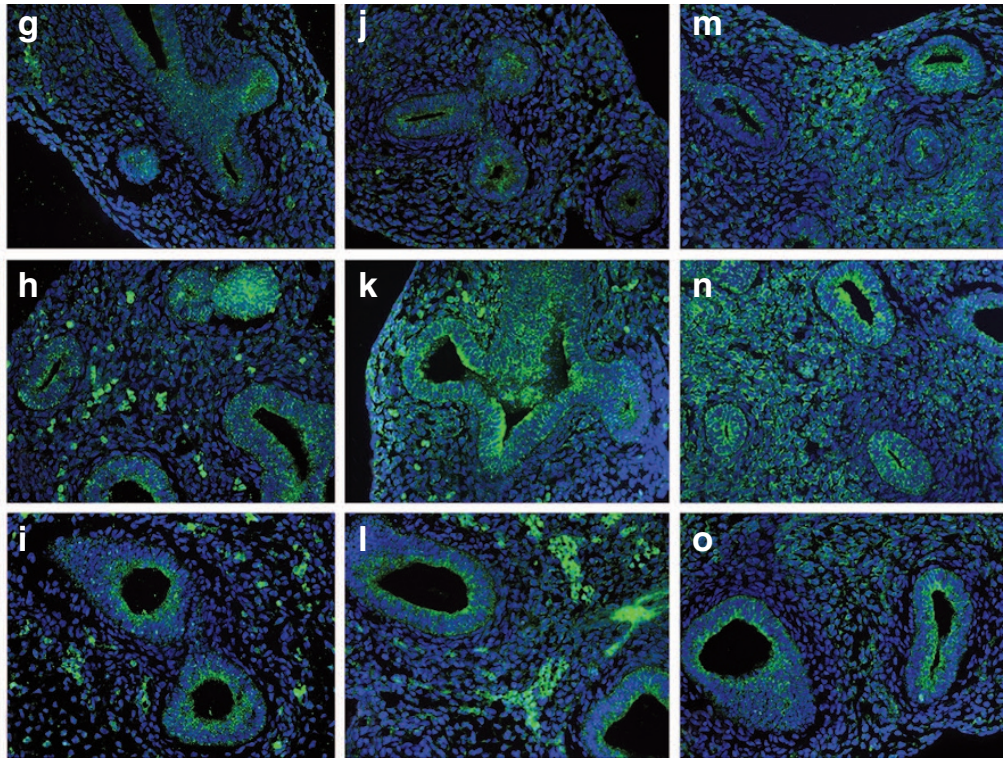

Figure 7. Immunofluorescence expression of the Shh signaling pathway in the pseudoglandular stage (E15) of lung development (Shh: (a) control, (b) adria EA-TEF (esophageal atresia and tracheoesophageal fistula), (c) adria noEA (original magnification $\times 10)$; Ptch: (d) control, (e) adria EA-TEF, (f) adria noEA (20x); Smo: (g) control, (h) adria EA-TEF, (i) adria noEA (20x); Gli2: (j) control, (k) adria EA-TEF, (I) adria noEA (20x); Gli3: (m) control, (n) adria EA-TEF, (o) adria noEA (20X)). Clear immunoreactivity was detected for Shh, Ptch, Smo, Gli2, and Gli3 protein in control, adria EA-TEF, and adria noEA groups. There were no significant differences concerning protein localization and all antibodies were mainly expressed in the epithelium, although Smo, Gli2, and Gli3 exhibited disperse immunoreactivity throughout the surrounding mesenchyme. Adria EA-TEF lungs showed an apparently increased signal for all the studied proteins.
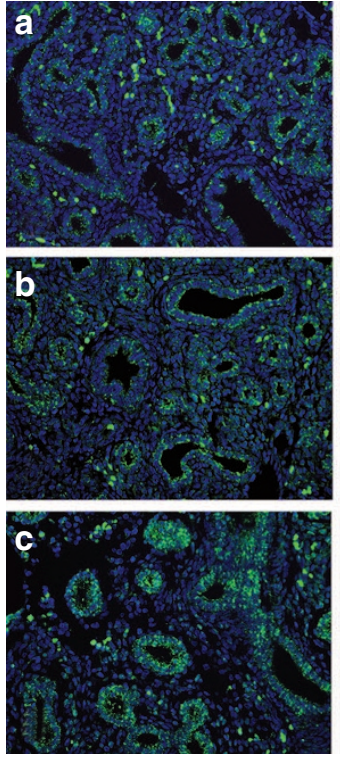
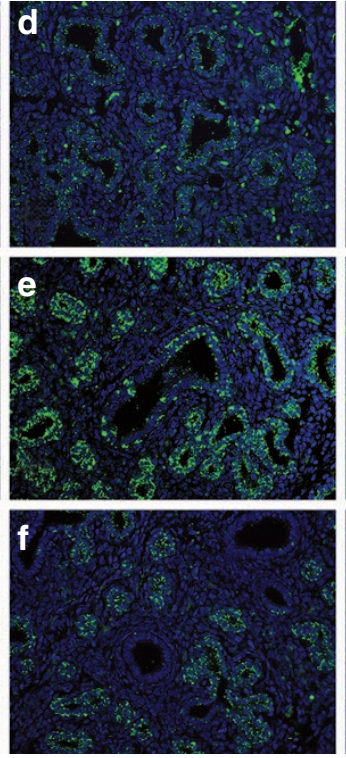
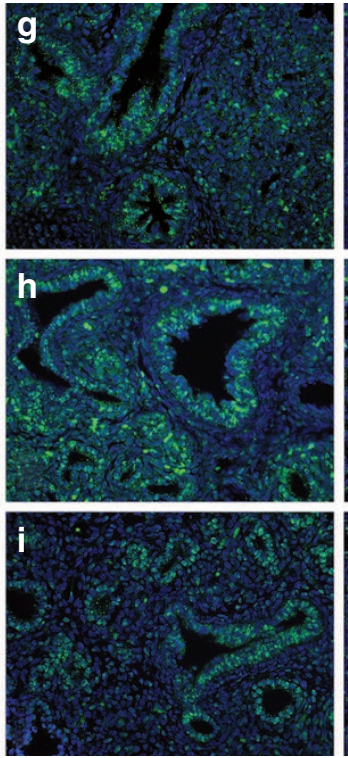
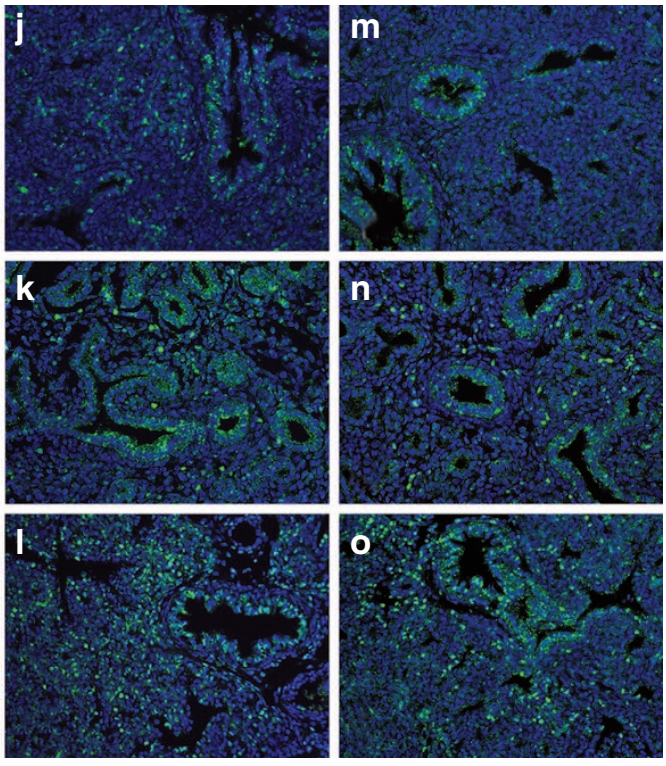

Figure 8. Immunofluorescence expression of the Shh signaling pathway in the canalicular stage (E18) of lung development (Shh: (a) control, (b) adria EA-TEF (esophageal atresia and tracheoesophageal fistula), (c) adria noEA (original magnification $\times 20)$; Ptch: (d) control, (e) adria EA-TEF, (f) adria noEA (20X); Smo: (g) control, (h) adria EA-TEF, (i) adria noEA (20X); Gli2: (j) control, (k) adria EA-TEF, (I) adria noEA (20x); Gli3: (m) control, (n) adria EA-TEF, (o) adria noEA (20X)). Clear immunoreactivity was detected for Shh, Ptch, Smo, Gli2, and Gli3 protein in control, adria EA-TEF, and adria noEA groups and there were no significant differences concerning protein localization. Adria EA-TEF lungs showed an apparently stronger epithelial and mesenchymal immunoreactivity for all the Shh pathway participants than the control group.

were somewhat different from those in the lungs of rats with EA-TEF. In fact, at E13 the expressions of Shh, Smo, Gli2, and Gli3 mRNA were higher than controls and much higher than in lungs of adria EA-TEF rats whereas Ptch showed similar levels of mRNA to the control group. Nevertheless, at the pseudoglandular stage, the expression levels of Shh, Ptch, Smo, and Gli2 were significantly higher than controls and similar to the adria EA-TEF lungs. It is interesting to point out that in the adriamycin-exposed embryos without EA-TEF, despite the upregulation of Shh until E18, there was a trend to reduce and 

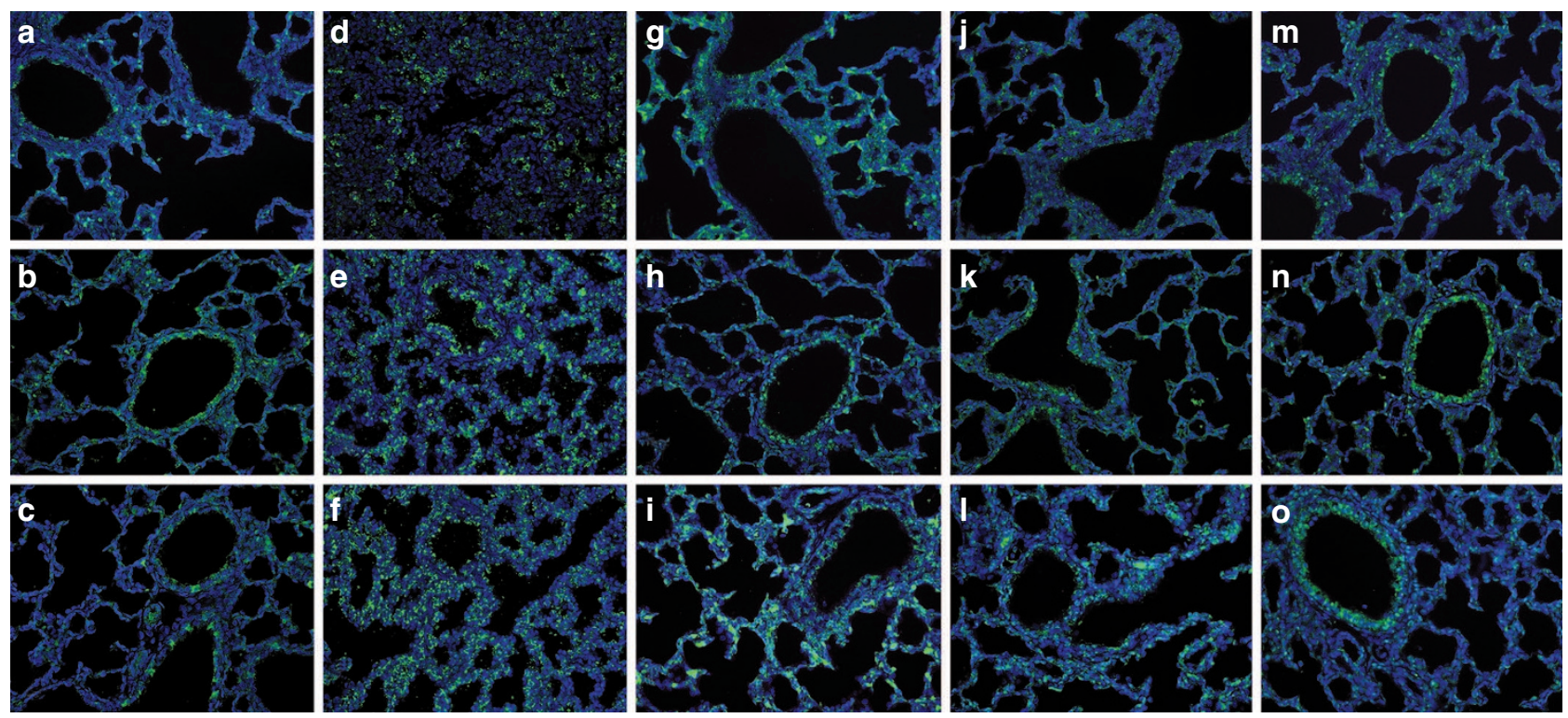

Figure 9. Immunofluorescence expression of the Shh signaling pathway in the saccular stage (E21) of lung development (Shh: (a) control, (b) adria EA-TEF (esophageal atresia and tracheoesophageal fistula), (c) adria noEA (original magnification $\times 20$ ); Ptch: (d) control, (e) adria EA-TEF, (f) adria noEA (20X); Smo: (g) control, (h) adria EA-TEF, (i) adria noEA (20x); Gli2: (j) control, (k) adria EA-TEF, (I) adria noEA (20x); Gli3: (m) control, (n) adria EA-TEF, (o) adria noEA (20X)). Clear immunoreactivity was detected for Shh, Ptch, Smo, Gli2, and Gli3 protein in control, adria EA-TEF, and adria noEA groups and there were no significant differences concerning protein localization. The adria EA-TEF lungs showed a slightly more intense expression of Shh, Ptch, Smo, Gli2, and Gli3 than controls.

normalize the expression throughout gestation. Furthermore, "erratic" behavior of the signaling pathway in these lungs suggests a disturbed pathway in some way different from the adria EA-TEF lungs eventually responsible for the different embryo phenotype induced by adriamycin administration (with or without EA-TEF). In addition, the putative effect of the tracheoesophageal fistula in lung organogenesis and growth must be considered, because Shh expression is also stimulated by physical pressures (26-28), and the disturbed swallowing plus the gastrointestinal obstruction seen in EA may perturb the lung dynamics exceptionally important for lung development.

In summary, and despite the absence of a semiquantitative protein assessment not allowing a discussion based on functional protein contents, immunofluorescence results seemed to be consistent with the real-time RT-PCR results. Both groups exposed to adriamycin showed a disturbed Shh signaling by real-time RT-PCR quantification. However, at the embryonic stage of lung development they expressed clear differences in mRNA levels of $S h h$ and its downstream components. In fact, in adria EA-TEF lungs, $S h$ is downregulated whereas in adria noEA lungs it is upregulated. This corroborates previous findings stating that a specific temporal expression of Shh is determinant for normal foregut and lung development. Shh disturbance induced by adriamycin is crucial for the development of EA-TEF, but seems to depend on gene gradient/dosage and other precise spatial and temporal interactions that would explain why only about $70 \%$ of rat fetuses from mothers exposed to adriamycin have EA-TEF (29).

Despite the major foregut anomalies seen in patients with EA-TEF/VACTERL association, the lung is, in general, grossly normal. However, up to $75 \%$ of EA-TEF patients suffer from
Table 1. Real-time RT-PCR probes and oligonucleotide primers

\begin{tabular}{|c|c|}
\hline Gene & Sequence $\left(5^{\prime}-3^{\prime}\right)$ \\
\hline \multicolumn{2}{|c|}{$\begin{array}{l}\text { Shh (UPL probe ENSRNOT00000008497. } \\
\text { 2|ENSRNOG00000006120.2) }\end{array}$} \\
\hline Forward & CAG GTG CACTGT GGCTGA T \\
\hline Reverse & CAC ATC CAC TGC TCT GTG AAA \\
\hline \multicolumn{2}{|l|}{ Smo } \\
\hline Forward & GCC TGA CTTTCT GCG TTG CA \\
\hline Reverse & GTT GTC TGT CCT CAC CAA GG \\
\hline \multicolumn{2}{|l|}{ Gli2 } \\
\hline Forward & TGA CTA CCT CAA CCC TGT GG \\
\hline Reverse & CTG TAG GTC AAG GCT GGC AT \\
\hline \multicolumn{2}{|l|}{ Gli3 } \\
\hline Forward & CGG AAT GGT TAC ATG GAG CC \\
\hline Reverse & GCA TCA ATC GGT ACA GGA GG \\
\hline
\end{tabular}

RT-PCR, reverse transcription polymerase chain reaction.

chronic respiratory symptoms and spirometric abnormalities apparently not related to gastroesophageal reflux, prematurity, or other EA-TEF sequelae (30). This may be explained by a deficient prenatal signaling, because there is increasing evidence that several signaling pathways important in prenatal development continue to have vital roles in adult life, namely in coordinating an appropriate response to injury (31).

In conclusion, our results demonstrate abnormal Shh pathway gene expression in adriamycin-exposed embryos since the embryonic stage of lung development may be due to the disturbed Shh environment previously described in the foregut of 
these embryos. From E15 onwards the lung seems to develop a molecular response aimed at catching up near-normal branching and growth by upregulation of the Shh signaling pathway. The significant differences between both groups exposed to adriamycin (with or without EA-TEF), particularly on E13, may elucidate the different phenotypes that arise from the adriamycin toxicologic rat model. The abnormal Shh signaling in lungs from rats with EA-TEF helps to improve knowledge on the lung organogenesis in this malformation, and may contribute to a better understanding of some unexplained chronic respiratory manifestations frequently present in human survivors to neonatal repair of EA-TEF.

\section{METHODS}

\section{EA-TEF Rat Model}

After IdiPaz Animal Care Committee approval (HULP PI-1501), time-dated pregnant Sprague-Dawley rats (OFA; Charles River Laboratories, Cerdanyola, Spain) were treated once a day at approximately the same hour and from gestational day 7 to 9 (the morning of sperm in the vaginal smear was considered as day 0) by i.p. injection of either $1.75 \mathrm{mg} / \mathrm{kg}$ Adriamycin (Farmiblastina Pharmacia, Madrid, Spain) or vehicle.

\section{Embryo Harvesting and Dissection}

Cesarean section was performed on E13, E15, E18, and E21, before killing the dams with an intracardiac injection of potassium chloride. At the elected time points, embryos were recovered, weighed, and dissected under a microscope to document the presence of EA-TEF. Lungs were harvested, weighed, photographed, and processed for RNA extraction (E13, E15, E18, and E21) and immunofluorescence staining (E15, E18, and E21).

Three groups of offsprings were compared: Control (C, $n=53$ ), adriamycin-exposed with EA-TEF (adria EA-TEF, $n=61$ ), and adriamycin-exposed without EA-TEF (adria noEA, $n=57$ ) from a minimum of four litters each.

\section{mRNA Extraction and CDNA Synthesis}

Total mRNA was isolated from snap-frozen lungs (at E13 three pools of 12 snap-frozen lungs were processed) using a High Pure RNA Tissue Kit (Roche Applied Science, Mannheim, Germany). Concentration and purity of RNAs were determined spectrophotometrically. RNA integrity was analyzed by $1 \%$ agarose gel electrophoresis; 20 ng of RNA/ $\mu$ l was retrotranscripted to complementary DNAs (cDNA) by reverse transcription reactions using a High Capacity RNA-to-cDNA Kit (Applied Biosystems, Carlsbad, CA). All cDNAs were stored at $-80^{\circ}$, until further use.

\section{Real-Time Reverse Transcriptase Polymerase Chain Reaction (Real-Time RT-PCR)}

Shh and Ptch lung expression was quantified in a LightCycler 480 with LightCycler 480 Probes Master (Roche Applied Science) for Shh (Roche Diagnostics, Mannheim, Germany) and Ptch (Rn01527980_ $\mathrm{m} 1$; Applied Biosystems) under the following conditions: $50{ }^{\circ} \mathrm{C}$ for $2 \mathrm{~min}, 95^{\circ} \mathrm{C}$ for $10 \mathrm{~min}$ followed by 40 cycles at $95^{\circ} \mathrm{C}$ for $15 \mathrm{~s}, 60^{\circ} \mathrm{C}$ for $20 \mathrm{~s}$, and $72^{\circ} \mathrm{C}$ for $1 \mathrm{~s}$.

Smo, Gli2, and Gli3 expression was quantified in a LightCycler 480 SYBR Green I Master (Roche Applied Science) using the primers described in Table 1. All RT-PCR reactions were run in duplicate in a total volume of $5 \mathrm{ng}$ of cDNA. The RT-PCR conditions were $95^{\circ} \mathrm{C}$ for $5 \mathrm{~min}$, followed by $50 \mathrm{cycles}$ at $95^{\circ} \mathrm{C}$ for $10 \mathrm{~s}, 60^{\circ} \mathrm{C}$ for $10 \mathrm{~s}$, and $72^{\circ} \mathrm{C}$ for $10 \mathrm{~s}$.

Results were normalized to the expression of $18 \mathrm{~S}$. The relative mRNA levels were determined by calculating the threshold cycle for all genes using the threshold cycle method.

\section{Immunofluorescence}

Lungs were fixed overnight in $4 \%$ paraformaldehyde. After inclusion in paraffin, $5-\mu \mathrm{m}$ sections were stained. Immunofluorescence staining was performed using standard techniques with anti-Shh antibody (Shh H-160; Santa Cruz Biotechnologies, Santa Cruz, CA), 1:50; anti-Ptch antibody (Ptch G-19; Santa Cruz Biotechnologies) 1:100; anti-Smo antibody (Smo N-19; Santa Cruz Biotechnologies) 1:50; anti-Gli2 antibody (49-617, ProSci, Poway, CA), and anti-Gli3 (49-600, ProSci) 1:50. Briefly, antigen recovery was performed with sodium citrate $(10 \mathrm{mmol} / \mathrm{l}, \mathrm{pH} 6)$ in microwave for $3-10 \mathrm{~min}$ followed by 2 -h incubation with $10 \%$ horse serum, $1 \%$ albumin TBS. Sections were incubated at $4{ }^{\circ} \mathrm{C}$ overnight with each of the primary antibodies then washed and incubated with universal secondary antibody (Vectstain Universal Quick Kit; PK-8800; Vector Laboratories, Burlingame, CA) for $1 \mathrm{~h}$, and finally with streptavidin Alexa Fluor 488 conjugate (S32354, Molecular Probes, Invitrogen, Carlsbad, CA) for $45 \mathrm{~min}$. The sections were mounted and the nucleus stained using Vectashield Mounting Medium for fluorescence with DAPI (H 1200; Vector Laboratories). Images were obtained from a minimum of six different slides from each group with a Leica LMD6000 fluorescence microscope (Leica Microsystems, Wetzlar, Germany).

\section{Statistical Methods}

Data are presented as mean \pm SD. Comparison between groups was performed with the Mann-Whitney $U$-test or the unpaired $t$-test where appropriate. The statistical significance level was set at $5 \%$.

\section{REFERENCES}

1. Orford J, Manglick P, Cass DT, Tam PP. Mechanisms for the development of esophageal atresia. J Pediatr Surg 2001;36:985-94.

2. Possögel AK, Diez-Pardo JA, Morales C, Navarro C, Tovar JA. Embryology of esophageal atresia in the adriamycin rat model. J Pediatr Surg 1998;33:606-12.

3. Arsic D, Cameron V, Ellmers L, Quan QB, Keenan J, Beasley S. Adriamycin disruption of the Shh-Gli pathway is associated with abnormalities of foregut development. J Pediatr Surg 2004;39:1747-53.

4. Mortell A, Gillick J, Giles S, Bannigan J, Puri P. Notable sequential alterations in notochord volume during development in the Adriamycin rat model. J Pediatr Surg 2005;40:403-6.

5. Vleesh Dubois VN, Quan Qi B, Beasley SW, Williams A. Abnormal branching and regression of the notochord and its relationship to foregut abnormalities. Eur J Pediatr Surg 2002;12:83-9.

6. Girosi D, Bellodi S, Sabatini F, Rossi GA. The lung and the gut: common origins, close links. Paediatr Respir Rev 2006;7:Suppl 1:S235-9.

7. Kim J, Kim P, Hui CC. The VACTERL association: lessons from the Sonic hedgehog pathway. Clin Genet 2001;59:306-15.

8. Ioannides AS, Copp AJ. Embryology of oesophageal atresia. Semin Pediatr Surg 2009;18:2-11.

9. Gillick J, Mooney E, Giles S, Bannigan J, Puri P. Notochord anomalies in the adriamycin rat model: a morphologic and molecular basis for the VACTERL association. J Pediatr Surg 2003;38:469-73; discussion 469-73.

10. Arsić D, Keenan J, Quan QB, Beasley S. Differences in the levels of Sonic hedgehog protein during early foregut development caused by exposure to Adriamycin give clues to the role of the Shh gene in oesophageal atresia. Pediatr Surg Int 2003;19:463-6.

11. Warburton D, Bellusci S, De Langhe S, et al. Molecular mechanisms of early lung specification and branching morphogenesis. Pediatr Res 2005;57(5 Pt 2):26R-37R.

12. Solomon BD. VACTERL/VATER Association. Orphanet J Rare Dis 2011;6:56.

13. Kenney AM, Cole MD, Rowitch DH. Nmyc upregulation by sonic hedgehog signaling promotes proliferation in developing cerebellar granule neuron precursors. Development 2003;130:15-28.

14. Marcelis CL, Hol FA, Graham GE, et al. Genotype-phenotype correlations in MYCN-related Feingold syndrome. Hum Mutat 2008;29:1125-32.

15. Kamachi Y, Kondoh H. Sox proteins: regulators of cell fate specification and differentiation. Development 2013;140:4129-44.

16. Williamson KA, Hever AM, Rainger J, et al. Mutations in SOX2 cause anophthalmia-esophageal-genital (AEG) syndrome. Hum Mol Genet 2006;15:1413-22. 


\section{Articles | Fragoso et al.}

17. Granata A, Savery D, Hazan J, Cheung BM, Lumsden A, Quaderi NA. Evidence of functional redundancy between MID proteins: implications for the presentation of Opitz syndrome. Dev Biol 2005;277:417-24.

18. Pinson L, Augé J, Audollent S, et al. Embryonic expression of the human MID1 gene and its mutations in Opitz syndrome. J Med Genet 2004;41:381-6.

19. Cohen MM Jr. Hedgehog signalling update. Am J Med Genet 2010;152A:1875-914.

20. Felix JF, de Jong EM, Torfs CP, de Klein A, Rottier RJ, Tibboel D. Genetic and environmental factors in the etiology of esophageal atresia and/or tracheoesophageal fistula: an overview of the current concepts. Birth Defects Res A Clin Mol Teratol 2009;85:747-54.

21. Whitsett JA. Disorders of lung morphogenesis. Paediatr Respir Rev 2006;7:Suppl 1:248.

22. Xiaomei L, Aras-Lopez R, Martinez L, Tovar JA. Lung hypoplasia in rats with esophageal atresia and tracheo-esophageal fistula. Pediatr Res 2012;71:235-40.

23. Fragoso AC, Aras-Lopez R, Martinez L, Estevão-Costa J, Tovar JA. Abnormal control of lung branching in experimental esophageal atresia. Pediatr Surg Int 2013;29:171-7.
24. Tovar JA. Stephen L. Gans distinguished overseas lecture. The neural crest in pediatric surgery. J Pediatr Surg 2007;42:915-26.

25. Bellusci S, Furuta Y, Rush MG, Henderson R, Winnier G, Hogan BL. Involvement of Sonic hedgehog (Shh) in mouse embryonic lung growth and morphogenesis. Development 1997;124:53-63.

26. Warburton D, El-Hashash A, Carraro G, et al. Lung organogenesis. Curr Top Dev Biol 2010;90:73-158.

27. Jesudason EC. Exploiting mechanical stimuli to rescue growth of the hypoplastic lung. Pediatr Surg Int 2007;23:827-36.

28. Cilley RE, Zgleszewski SE, Chinoy MR. Fetal lung development: airway pressure enhances the expression of developmental genes. J Pediatr Surg 2000;35:113-8; discussion 119.

29. Fragoso AC, Martinez L, Estevão-Costa J, Tovar J. Maternal hyperthyroidism increases the prevalence of foregut atresias in fetal rats exposed to adriamycin. Pediatr Surg Int 2014;30:151-7.

30. Legrand C, Michaud L, Salleron J, et al. Long-term outcome of children with oesophageal atresia type III. Arch Dis Child 2012;97:808-11.

31. Lees C, Howie S, Sartor RB, Satsangi J. The hedgehog signalling pathway in the gastrointestinal tract: implications for development, homeostasis, and disease. Gastroenterology 2005;129:1696-710. 